\title{
The Real-Time Processing of Sluiced Sentences
}

\author{
Josée Poirier • Katie Wolfinger • Lisa Spellman • \\ Lewis P. Shapiro
}

Published online: 13 March 2010

(c) The Author(s) 2010. This article is published with open access at Springerlink.com

\begin{abstract}
Ellipsis refers to an element that is absent from the input but whose meaning can nonetheless be recovered from context. In this cross-modal priming study, we examined the online processing of Sluicing, an ellipsis whose antecedent is an entire clause: The handyman threw a book to the programmer but I don't know which book the handyman threw to the programmer ellipsis. To understand such an elliptical construction, the listener arguably must 'fill in' the missing material ("the handyman threw to the programmer") based on that which occurs in the antecedent clause. We aimed to determine the point in time in which reconstruction of the sluiced sentence is attempted and whether such a complex antecedent is re-accessed by the ellipsis. Out of the two antecedent constituents for which we probed, only the Object (programmer) was found active in the elliptical clause, confirming that an antecedent is attributed to the sluice in real time. Possible reasons for the non-observation of the Subject (handyman) are considered. We also suggest that ellipses are detected earlier in coordinated than subordinated sentences.
\end{abstract}

Keywords Sluicing $\cdot$ Ellipsis $\cdot$ Complexity $\cdot$ Sentence processing

\section{Introduction}

The term Ellipsis refers to a common family of constructions in which a part is unpronounced. For example, in sentence [1], no verb is present in the second clause (the ellipsis is represented by $\varnothing$ ), yet comprehenders readily understand that Luis likes brownies:

J. Poirier · L. P. Shapiro

SDSU/UCSD JDP Language and Communicative Disorders, San Diego, CA, USA

J. Poirier $(\varangle) \cdot$ K. Wolfinger · L. Spellman · L. P. Shapiro

Speech, Language, and Hearing Sciences (SLHS), San Diego State University, Bldg \#221,

5500 Campanile Drive, San Diego, CA, 92182-1518, USA

e-mail: josee.phd@gmail.com 
[1] Jillian likes apple pie and Luis, $\varnothing$ brownies.

To understand an elliptical sentence, the content of the elided or silent material must be recovered from an earlier part of the sentence, the antecedent (underlined in [1]). Ellipsis is subject to a Parallelism constraint that forces the elliptical clause (EC) to parallel the antecedent clause (AC). This constraint ensures that the elided material is correctly reconstructed as the verb 'likes' in [1], and not 'hate', for example. The exact nature of the parallelism requirement (semantic, syntactic or discourse-based) extends beyond the scope of this paper. For now, it suffices to note the intimate relation between the antecedent and ellipsis clauses.

The listener's ability to readily and unambiguously understand ellipsis is nothing short of remarkable: the input incompletely represents the message to convey. The burden is thus put on the listener to somehow detect the 'missing' pieces and reconstruct the intended meaning in a matter of milliseconds. Thus the comprehension system must recover the unpronounced message by finding and retrieving an antecedent that is long gone in the temporal stream by the time the ellipsis is processed.

Unveiling the mechanism by which meaning is assigned to an ellipsis has been the focus of several psycholinguistic studies. In an early proposal, Frazier and Clifton (2000) suggested that an antecedent-along with its semantic content and syntactic structure-is copied into the elliptical clause. According to this account, the copying operation induces higher processing costs for more complex antecedents, as more structure needs to be copied. However, several studies have reported that the complexity of the antecedent does not affect ellipsis processing (Frazier and Clifton 2001, 2000; Martin and McElree 2008). For example, Martin and McElree argued that ellipsis does not involve syntactic reconstruction; instead, a content-addressable pointer mechanism directs the processor to the mnemonic representation of the antecedent. As a response to the absence of complexity effects, Frazier and Clifton $(2001,2005)$ have proposed two potential mechanisms to resolve the ellipsis: a cost-free copy operation (i.e., building structure is computationally free or cheap) and a structure-sharing process (i.e., the ellipsis and antecedent share a syntactic representation). Importantly, syntactic structure is assumed to be present at the elision site under these proposals (see Frazier and Clifton 2005, for arguments in support of this assumption).

Once an ellipsis is detected, its antecedent is identified, retrieved from memory and integrated into the elliptical clause. The processor does not wait until the end of the sentence to resolve the ellipsis; it establishes the antecedent-ellipsis link as the sentence unfolds. Support for the immediacy of establishing the link comes from several cross-modal priming studies that demonstrated that elided material is recovered in real time at the elision site (Poirier and Shapiro, under revision; Shapiro and Hestvik 1995; Shapiro et al. 2003). For example, Shapiro et al. found that priming — an index of lexical activation — was obtained for the direct object tie at the did too:

[2] The mailman [bought a tie $]_{\mathrm{VP}}$ and the fireman did $\varnothing$ too according to the salesclerk.

Because no priming was observed shortly before the elided position, it was concluded that processing the ellipsis triggered the re-activation of the antecedent. In contrast, the antecedent clause's subject (mailman) was not re-accessed in the EC, indicating that only the syntactically-defined antecedent of the ellipsis (a verb phrase in [2]) is recovered at the elision site (Shapiro et al. 2003; Poirier and Shapiro, under revision). In other words, because the VP does not include the subject, mailman is not observed at the elision site. This evidence is irreconcilable with the claim that antecedent-external elements are also re-accessed during the reconstruction of the elided material (Koeneman et al. 1998). On the other hand, this pattern is directly in line with the literature on anaphoric relations, in which an element 
(e.g., a pronoun) seeks an antecedent. The referent-seeking pronoun triggers the immediate reactivation of the antecedent; in cases where multiple options are available, only the syntactically legal antecedent is re-accessed (Nicol 1988). Together, these findings indicate that syntactic constraints aid the processor to connect two nonadjacent co-referents by guiding the selection and retrieval of the appropriate antecedent.

If restrictions prevent the inclusion of extraneous phrases in the reconstruction of the elided material, it is unknown whether all components within the lawful antecedent are recovered. Previous studies have only investigated the re-activation of smaller antecedents (noun phrases in anaphora, arguments within the verb phrase in verb-phrase ellipsis). In some cases, however, the material to recover is more complex: for instance, Sluicing - the elliptical structure this study examined-involves the elision of an entire clause (IP):

[3a] The fifth-grader doesn't like snakes but his mother doesn't know why $\varnothing$.

[3b] The fifth-grader doesn't like snakes but his mother doesn't know why the fifth-grader doesn't like snakes.

To comprehend [3a], the sluiced sentence (strikethrough in [3b]) must be recovered. To do so, an entire clause needs to be re-accessed from memory.

On the other hand, the antecedent in Sluicing shows variability that can be exploited to examine the mechanisms underlying ellipsis resolution; the phrases composing the antecedent clause (the IP) can have many properties. For example:

[4a] (The secretary typed something) IP, but I don't know what $\varnothing$ exactly.

[4b] (The secretary typed somewhere) $)_{\text {IP }}$, but I don't know where $\varnothing$ exactly.

[Examples from Dickey and Bunger, under review]

In these examples, the sluiced sentence needs to be recovered from the previous clause. Despite the fact that both antecedents are IPs, they differ in one aspect, the syntactic function of the postverbal Noun Phrase (something vs. somewhere). In [4a], the NP is an argument of the verb, whereas the NP is an adjunct in [4b] (only arguments are required for grammaticality-adjuncts are superfluous information). This distinction has processing consequences: Dickey and Bunger (under review) found that the elliptical clause is read more slowly in the case of adjunct elision [4b]. Furthermore, they observed similar processing costs in the non-elided versions of these sentences, suggesting that the same operation applies to both types of sentences. This finding suggests that the sluiced sentence is reconstructed with a syntactic structure similar to its non-elided version.

Similarly, the mechanism underlying the reconstruction of sluiced sentences whose antecedent is incomplete can be investigated. Consider:

[5a] [The secretary typed something] IP, but I don't know what $\varnothing$ exactly.

[5b] [The secretary typed quickly $]_{I P}$, but I don't know what $\varnothing$ exactly.

[Examples from Dickey and Bunger, under review]

The sluiced sentence in [5a] is reconstructed where something and what co-refer to the object typed. In contrast, in [5b], the processor is faced with a constituent of the sluiced sentence (what) that has no explicit counterpart in the antecedent. The 'incompleteness' of the antecedent phrase results in longer processing times in [5b] than in [5a] (Dickey and Bunger, under review; Frazier and Clifton 1998). This processing cost seems to stem from a general penalty for non-parallelism between clauses and not from an ellipsis-specific operation to create the missing syntactic position for what in the sluiced sentence (Chung et al. 1995; Dickey and Bunger, under review). 
This literature on the processing of ellipsis is important because it can illuminate several aspects of a general sentence processor, including the role of dependency relations and parallelism (see above), focus (see, for example, Carlson et al. 2007; Frazier and Clifton 1998), and the role of preference and lexical information during parsing (Shapiro et al. 2003). Yet, there is a void in this literature, one that we hope to fill with the present effort and one where Sluicing can help: Little is known about the nature of the material that is reconstructed in ellipsis other than in VPE. Sluicing offers the opportunity to examine whether multiple DPs (from an IP or clause) are reconstructed from the antecedent. In the Dickey and Bunger effort, for example, a self-paced moving window task was used, and distinctions based on reading times in the ellipsis clause were indicative of processing information from the antecedent clause. In the present effort we use another well-tested method-priming — to establish the basic activation patterns in sluiced sentences. We predict that Sluiced sentences will be processed similarly to Verb-Phrase Ellipsis and trigger the re-activation of their licit antecedent, a full clause.

Furthermore, we aim to clarify the point in time at which the ellipsis is resolved. As previously mentioned, the antecedent is re-activated at the temporal location of the ellipsis (at 'did too' in Verb-Phrase Ellipsis; Shapiro and Hestvik 1995). In the same study, Shapiro and Hestvik reported a delay in re-accessing the antecedent in the same sentences when conjoined by the conjunction 'because' compared to 'and'. The authors hypothesized that the establishment of a cause-effect relation between conjuncts in 'because'-conjoined sentences protracted the resolution of the ellipsis. A corollary is that ellipsis resolution takes place earlier or faster in 'and'-conjoined sentences. Therefore, the use of an alternate conjunction, may it require the establishment of a cause-effect relation or not, would be responsible for the later effects. We test this hypothesis in the current study by conjoining our elliptical sentences with a third conjunction that does not imply a cause-effect relation: 'but'. This hypothesis thus predicts antecedent re-activation downstream from the temporal location of the ellipsis with the present stimuli.

In short, this experiment was designed to establish two facts about the processing of Sluicing: how much of the antecedent is recovered in the elided clause and when does ellipsis resolution take place in sentences not conjoined by 'and'. To do so, activation of the Subject and Object of the antecedent clause was probed at the temporal point of the sluice and also 'downstream' from the sluice, using a cross-modal priming paradigm.

\section{Experiment}

\section{Participants}

Thirty students from San Diego State University (mean age: 22.3; range: 18-48) participated in this experiment for course credit. All participants were monolingual native speakers of English with normal or corrected-to-normal auditory and visual acuity. Participants had not been exposed to a second language before the age of six and had no history of neurological injury or learning disorder.

Materials

Forty sentences containing a sluice (strikethrough) such as [6] were created:

[6] The handyman threw a book to the programmer but I don't know which book [the handyman threw to the programmer] and no one else seems to know. 
The sentences matched the following template: NP (subject)—verb-NP (non-sluiced object)—NP (sluiced object)—'but I don't know which (non-sluiced object)—closing segment starting with 'and'. The closing segment was incorporated to offer ample time to test at a position downstream from the location of the sluice (offset of book) while avoiding end-ofsentence wrap-up effects (Balogh, Zurif, Prather, Swinney, and Finkel 1998). Importantly, no semantic relatedness was allowed within the sentences to prevent confounding priming effects.

The antecedent of the ellipsis (namely, the sluiced sentence) was the first clause in all sentences, minus the non-sluiced object (e.g.: the handyman threw_to the programmer). We probed for the sluiced subject (handyman) and object (programmer) in the ellipsis clause; these represent the left and right edges of the antecedent clause. We chose not to probe for the verb as previous work has suggested that predicates remain activate throughout clauses (Callahan, Shapiro, and Love, submitted; De Goede et al. 2010).

Due to the nature of the stimuli, which required both a direct and an indirect object (1 sluiced, 1 nonsluiced), distinct sentences were used to probed for the Object and for the Subject. In effect, only a small number of English verbs imperatively take two objects. Thus, the number of tokens that could meet the double-object requirement was limited $(n=20)$. These tokens were dedicated to the examination of the re-activation of the antecedent Object. Twenty additional sluiced sentences were designed to study the re-activation of the antecedent Subject. In this way, the sample of sluiced sentences was doubled to forty and allowed for more confidence inferring over the population of Sluicing constructions.

In addition to the test tokens, 85 fillers were generated. Twenty tokens were non-elliptical sentences that approximated the structure of the test sentences, while the other 65 were nonelliptical constructions that varied in syntactic structure. In addition to these items, 41 yes/no questions per session were composed. The questions were presented at irregular intervals with the goal of encouraging attention to the sentences. Finally, an additional ten sentences and two questions were created to form a practice session. In short, less than $25 \%$ of the 178 sentences composing a session were test items.

Visual probes were assigned to each of the sentences. For the filler sentences, roughly half of the visual probes were English pseudowords; the presentation of these probes was randomly dispersed over the course of the sentences so as to prevent participants from predicting when the probes would appear. For test sentences, probes were English words that were semantically related to either the antecedent's subject (sentences S1-S20, see Appendix) or to the antecedent's object (sentences S21-S40, see Appendix). The test probes were, but for one exception, the first semantic associate of the noun of interest (Subject or Object). ${ }^{1}$ These probes were presented at specific points in time: at the elision site (1) below) and at a downstream point ( $500 \mathrm{~ms}$ later; approximately at (2) below):

[7] The handyman threw a book to the programmer but I don't know which book (1) and no one, (2) else seems to know.

These probe positions were determined based on the temporal location of the sluice (1) and on previous findings that ellipsis is detected and interpreted some $500 \mathrm{~ms}$ downstream from the elision site in subordinated sentences (Shapiro and Hestvik 1995). Since the test sentences in this experiment were constructions conjoined by 'but', it was expected the sluice would trigger the re-activation of its antecedent after a certain delay (that is, at (2)). Hence, probe position 1 served as a baseline to ensure the antecedent had decayed in activation before being re-activated by the ellipsis.

1 The exception was the pair doctor-nurse, where nurse is the third associate of doctor. We thank Dr. Sarah Callahan for sharing her experimentally-built database of nouns and their semantic associates. 


\section{Design}

There were eight conditions, with noun of interest (Subject, Object), probe position (PP1, PP2) and probe type (Control, Related) as factors. The complete design was counterbalanced across four lists: each test sentence appeared in every condition but for the factor Noun of Interest, which was a between-sentence factor. Participants came in for two sessions, 1 week apart, and were randomly assigned a pair of scripts: in the first session, a sentence was presented at one probe position with an Object- or Subject-related probe; in the second session, the same sentence appeared at the same position but with its control probe. Within a session, test and filler sentences were ordered pseudo-randomly following the constraint that no more than three items with similar properties (filler or test, noun of interest, probe position or type, word/nonword probe) would be sequentially presented. In sum, each sentence was heard only once per session, but twice in total. Importantly, participants contributed data to all conditions albeit with different sentence/condition combinations.

\section{Procedure}

A Cross-Modal Lexical Priming paradigm was used. The sentences were digitally recorded at a normal speech rate and were aurally presented through headphones to participants who were sitting in a soundproofed booth facing a computer. At a pre-determined point during the unfolding of the sentence (see Section 1.2.2), a visual probe appeared centrally on the screen for $300 \mathrm{~ms}$. Participants were asked to perform two tasks: 1) to indicate quickly and accurately whether the visual probe was a word of English using a two-button box; 2) to carefully listen to the sentences and answer a comprehension question when prompted.

A session began with a series of practice items. When participants showed comprehension of the tasks at hand, the experiment began. Presentation software was used to present the auditory and visual stimuli and to record participants' accuracy and reaction times with millisecond accuracy.

\section{Results}

Incorrect responses ("nonword" to a real word test stimulus or failure to respond in the allotted time) were excluded, which represented $4.9 \%$ of the data. Two participants did not perform satisfactorily on the lexical decision task, averaging less than $60 \%$ correct responses. Due to their demonstration of insufficient attention and/or comprehension of the task, these participants were excluded from further analysis.

Data from correct responses were compiled. As is standard in the analysis of such data, a cutoff was applied whereby reaction times (RTs) faster than $300 \mathrm{~ms}$ or slower than $1,500 \mathrm{~ms}$ were excluded ( $1.5 \%$ of data). The observation of $\mathrm{RT}_{\text {related }}<\mathrm{RT}_{\text {control }}$ reflects a priming effect that is interpreted as evidence that the prime (i.e., the noun phrase of interest, Subject or Object) was activated. Table 1 presents the conditional means and standard deviations for each noun phrase (NP). As can be seen in Fig. 1, priming was obtained for the Object at the downstream position. No reliable effect was observed for the Subject.

An Analysis of Variance (ANOVA) was performed with subjects $\left(F_{1}\right)$ and items $\left(F_{2}\right)$ as random variables and with Noun of Interest (NP; Subject, Object), Probe Position (PP; PP1, PP2) and Probe Type (PT; control, related) as within-subject factors (with the exception of Noun of Interest, which was a between-item factor in the F2 analyses). 
Table 1 Mean reaction times and standard deviations to related and control probes for the Subject and the Object at each probe position

\begin{tabular}{lllllll}
\hline NP & Data & \multicolumn{2}{l}{ PP1 (Elision site) } & & \multicolumn{2}{l}{ PP2 (Downstream) } \\
& & Control & Related & & Control & Related \\
\hline Subject & Mean (SD) & $711(118)$ & $703(127)$ & & $719(124)$ & $727(123)$ \\
Object & Mean (SD) & $715(108)$ & $719(125)$ & & $748(125)^{\mathrm{a}}$ & $711(110)^{\mathrm{a}}$ \\
\hline
\end{tabular}

a control/related conditions statistically different

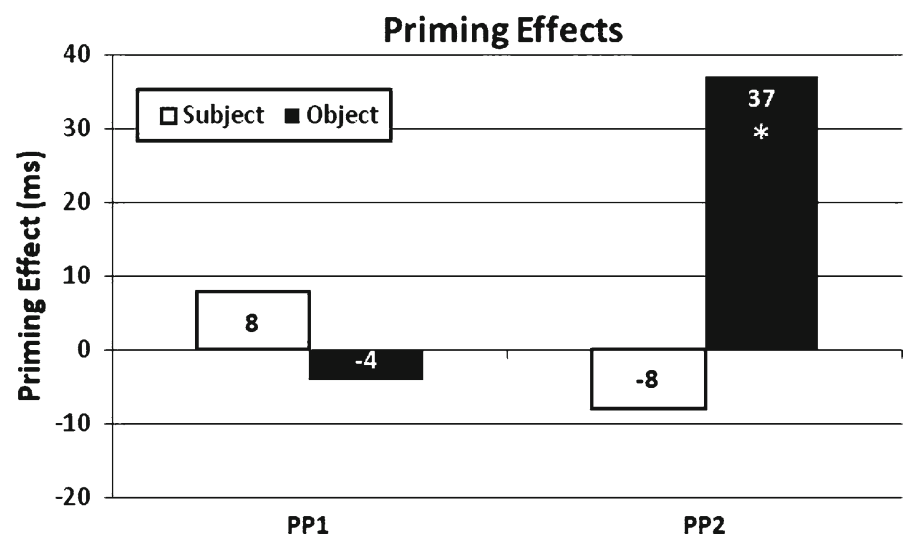

Fig. 1 Priming effects (control minus related RTs) for the Subject and the Object at each probe position. * statistically significant effect

A main effect of Probe Position $\left(F_{1}(1,27)=5.29, p<0.05\right)$, as well as a main effect of Probe Type $\left(F_{2}(1,19)=3.44, p=0.079\right)$ were observed. A marginally significant threeway interaction between NP, Probe Position and Probe Type was also revealed $\left(F_{1}(1,27)=\right.$ $3.49, p=0.07)$. To explore the nature of this interaction, 2-way ANOVAs were performed for each NP separately. For Object, a near-significant Probe Position $\mathrm{x}$ Probe Type interaction was found $\left(F_{1}(1,27)=3.25, p=0.08\right)$. No main effect or interaction was observed with Subject.

We also computed one-tailed planned comparisons to directly address test the prediction of late re-activation of the Object and of the Subject. Our expectations were partially supported: Object-related probes were indeed responded to faster than control probes at the downstream position (subject-based: $t_{27}=2.30, p<0.05$; item-based: $t_{19}=2.20, p<0.05$ ), and not at the elision site (subject-based: $t_{27}=-0.25, p>0.05$; item-based: $t_{19}=0.05, p>0.05$ ). However, for Subject, the priming effect was not statistically significant at the elision site (subject-based: $t_{27}=0.55, p>0.05$; item-based: $t_{19}=0.60, p>0.05$ ) or at the expected downstream position (subject-based: $t_{27}=-0.53, p>0.05$; item-based: $t_{19}=-0.30, p>$ $0.05) .^{2}$

\footnotetext{
${ }^{2}$ We ran an additional experiment to determine if the Subject of the antecedent, due to its position in the sentence (first noun phrase), could have been too difficult to identify properly by the participants (perhaps because of a lack of attention so early on in the sentence), which would have made it impossible for them to retrieve the noun phrase later in the sentence. We also aimed to confirm that our Subject-related probes could yield priming effects, that is, that they were close semantic associates of the prime (the Subject). Briefly, participants who showed object activation in the ellipsis clause also showed priming for the subject at the point where the subject was first encountered in the antecedent clause, suggesting that, in principle, the subject
} 


\section{Discussion}

The current study extended the examination of elliptical sentences to Sluicing. In Sluicing constructions, a constituent is unpronounced (strikethrough below):

[The handyman threw a book to the programmer] $]_{\mathrm{AC}}$ but I don't know which book [the handyman threw to the programmer] EC.

To be understood, sluiced sentences must be interpreted based on the antecedent clause (AC; squared brackets above). The purpose of the study was to determine if the antecedent clause is re-activated in the elliptical clause (EC) and if so, at what point in time. Our study revealed that processing a sluiced sentence does trigger re-access of its antecedent. Yet unexpectedly, the antecedent clause was not re-activated in its entirety: namely, only priming for the Object (e.g., programmer), and not the Subject (e.g., handyman) was obtained.

We first turn to the observed re-activation of the Object in the elliptical clause. This finding is directly in line with the literature on ellipsis and on anaphora where it has been repeatedly demonstrated that antecedent re-activation occurs as soon as the referent-seeking element is processed (e.g., Nicol 1988; Shapiro and Hestvik 1995; Swinney et al. 1987).

Moreover, the current study determined for the first time the exact timing of the ellipsis detection/antecedent re-access in Sluicing: the processing system postulates the existence of the elided clause after its temporal position in the sentence (namely, at the offset of which book). The delay in antecedent re-activation observed in the present study appears to reflect the necessity to infer the ellipsis from sentential context. In effect, there are no overt cues hinting to the upcoming presence of an ellipsis or to its actual location. Therefore, the silent phrase goes unnoticed until the processing of the post-elision segment, where the system reacts to a missing constituent (namely, the elided phrase).

Nonetheless, previous priming studies of Verb-Phrase Ellipsis have repeatedly found antecedent re-access at the temporal location of the ellipsis (Shapiro and Hestvik 1995, Exp. 1; Shapiro et al. 2003; Kaan et al. 2004; Poirier and Shapiro, under revision). If a delay is required to infer the ellipsis from context, how can effects be observed this early? The answer, we believe, is that ellipsis resolution timing is affected by the type of conjunction used in the sentences. In effect, ellipses that are posited earlier appear in and-coordinated constructions (e.g., Verb-Phrase Ellipsis studies mentioned above); ellipses resolved after a delay surface in constructions otherwise conjoined (by 'but' or 'because'; Shapiro and Hestvik 1995, Exp.2; the present study). How could the presence of the conjunction 'and' expedite the postulation of an ellipsis?

It has been shown that the conjunction 'and' induces expectations of parallelism (structural similarity) between conjuncts (Knoeferle 2007). A considerable amount of evidence has accumulated on the effect of Parallelism in nonelliptical clauses; many studies have reported faster processing of a second conjunct following a parallel, first conjunct (Apel et al. 2007; Frazier et al. 2000, 1984; Knoeferle and Crocker, in press; Mauner et al. 1995; Pickering et al. 2000). Thus, parallelism may function as a heuristic used by the processing system to facilitate parsing (see Bock 1986 and Pickering and Ferreira 2008 for a similar effect in production). However, this facilitation effect is limited to coordinated sentences that are joined by parallelism-implying conjunctions, such as 'and' and 'while', but not 'but' (Knoeferle 2007). Returning to ellipsis, ellipsis resolution has been observed early in

Footnote 2 continued

could be activated, and that our probes were well-controlled and could not have accounted for our lack of subject effects in the ellipsis clause. Thus, the lack of effect of subject activation in the ellipsis clause appears to be a robust, if null, result. 
'and'-conjoined sentences, and later in sentences otherwise conjoined (by 'because' or 'but'). It thus seems that the expectation of parallelism in 'and'-coordinated sentences speeds up the postulation and resolution of ellipsis. Two mechanisms could potentially underlie such facilitation: the expectation of parallelism can render the antecedent already accessible at the temporal location of the ellipsis by re-activating it before the ellipsis is encountered. In fact, studies have reported activation of first-conjunct material as soon as the conjunction 'and' has been processed (Callahan et al., submitted; Poirier and Shapiro, under revision). Alternatively, the processor could attempt to parse the second conjunct identically to the first; at the point in time where the structure differs from the first clause (that is, at the temporal location of the ellipsis), an elided constituent would immediately be postulated. Regardless of the mechanism at play, the time course of ellipsis resolution seems to be tied to the type of conjunction joining the elliptical and antecedent clauses.

Returning to the observation of Object reactivation in the present study, this finding seems to indicate that the antecedent is re-accessed in Sluicing much like other anaphora (verbphrase ellipsis, pronouns, traces, etc). By contrast with verb-phrase ellipsis, however, the antecedent recovered was not, strictly speaking, the syntactically-defined antecedent. In effect, no reliable evidence of Subject reactivation was obtained in the elliptical clause. Thus, it cannot be confirmed from these results that the entire antecedent is reconstructed in Sluicing. Any tentative conclusion is based on a null result (i.e., absence of Subject activation), but we cautiously consider possible explanations for this apparent partial antecedent recovery.

So, why would the Subject not be re-activated in the elliptical clause, especially considering the potentially adverse effect of only partially recovering the meaning of the elided constituent might have on comprehension? One possibility is that the Subject NP may be too far back in the temporal stream (due to its sentence-initial position) for it to be successfully recovered. This possibility is not strongly supported experimentally. For example, in nonelliptical sentences, Subject re-activation was observed, despite about 26 syllables separating the sentence-initial Subject and its reactivation site (Friedmann et al. 2008). In Ellipsis, previous studies using the speed-accuracy trade-off paradigm demonstrated that the distance between an ellipsis and its antecedent does not increase the processing load associated with antecedent retrieval (Martin and McElree 2008). Relatedly, antecedent length did not affect interpretation accuracy or speed (Martin and McElree 2008). Taking into consideration that the maximal Subject-Ellipsis distance and antecedent length tested in the work above mentioned by far exceeded those in the current study, it is unlikely that the Subject here was too far from the ellipsis to be retrieved later in the sentence.

Another possibility regards the Subject's syntactic role in the antecedent clause. Subjects are external arguments of the verb (and objects, internal arguments). Very little psycholinguistic work has investigated the external-internal argument distinction. Linguistic analyses have argued that external arguments are not selected by the verb (in contrast with internal arguments), but rather by the entire verb phrase (the phrase consisting of the verb and its object; e.g.: Marantz 1984). Moreover, several languages such as Spanish, Italian, Arabic, Finnish, Chinese, Japanese and Hindi (among others) allow for the absence of overt subjects in sentences, whereas only a handful permit the same for objects. External arguments thus seem more disposable than internal ones; the parser could disregard the 'superfluous' Subject NP when reconstructing the ellipsis. This argument, however, is inconsistent with studies that found subject/external argument re-activation in anaphoric relations (Friedmann et al. 2008; Nicol 1988; Zurif et al. 2003; Bever and Sanz 1997; among others), including in elliptical constructions (Poirier and Shapiro, under revision; Koeneman et al. 1998). Hence, the function of the external argument does not seem to preclude re-activation of a noun phrase. Nonetheless, it cannot be ruled out that the complexity of the antecedent in Sluicing imposes 
restrictions on the parser that are otherwise absent. For instance, it is conceivable that the parser, in a need for economy and faced with high processing loads, would elect to drop a phrase that is dispensable- the external argument.

We thus now turn to the possibility that antecedent complexity affects antecedent retrieval. Namely, it may be the case that in Sluicing, the 'grain size' of the antecedent prevents full antecedent reconstruction (at least online). This possibility would be consistent with recent findings from aphasia that suggest antecedent complexity may increase the burden on the processing system. In effect, anterior-lesioned Broca's patients can retrieve noun phrases to assign to referent-seeking elements (albeit after a delay; Love et al. 2008), but fail to re-activate an antecedent verb phrase in verb-phrase ellipsis (Poirier et al. 2009). Considering that verb phrases represent more material and structure than noun phrases, it is possible that the amount of material to retrieve overwhelms the processing system of these patients. ${ }^{3}$ However, speed-accuracy trade-off studies of ellipsis report that antecedent complexity affects neither speed nor accuracy (with one exception: a higher number of discourse referents seem to decrease accuracy levels; Martin and McElree 2008). Furthermore, other studies have repeatedly reported that antecedent complexity does not increase processing loads in ellipsis (Frazier and Clifton 2000, 2001). Hence, the support for the view that antecedent complexity affects ellipsis resolution is equivocal.

Nonetheless, we believe the issue of complexity should be further scrutinized. For one thing, the conclusion that antecedent complexity does not affect antecedent retrieval is based on a null result (i.e., the absence of increased reaction times with more complex antecedents). It is thus possible that there are indeed complexity effects, but that they have thus far been missed. One reason complexity effects might not have been detected could involve methodological considerations. In effect, complexity effects have only been studied in the written modality (in self-paced reading, speed-accuracy tradeoff and eye-tracking paradigms) and in stimuli where the ellipsis is sentence-final (Frazier and Clifton 2000, 2001; Martin and McElree 2008). These paradigms might have been unable to detect complexity effects, perhaps due to the modality or the presentation style. For example, all previous studies on complexity effects presented their stimuli in a segmented, phrase-by-phrase style that arguably chunks the input unnaturally. This chunking process, might, in turn, facilitate the retrieval of large amounts of information. In addition, these studies investigated ellipsis in sentence-final positions. Considering that end-of-sentence wrap-up effects have been shown to trigger reactivation of previously encountered material (Balogh et al. 1998), it is possible that processing costs incurred by the antecedent retrieval operation could have been masked by ellipsis-unrelated, wrap-up activation processes. On the other hand, the present study aurally presented the sentences in a continuous fashion, and the stimuli were carefully constructed so that the ellipsis would not appear sentence-finally. If any of these methodological factors prevented the detection of (potentially minute) complexity effects in previous experiments, the partial antecedent reconstruction found in the present study could, in principle, reflect complexity effects.

Along the same lines, complexity effects might exist if the definition of complexity is reconsidered. For example, there are two factors that do not increase processing loads, but that negatively affect ellipsis interpretation: distance and number of referential noun phrases (NPs). Speed-accuracy trade-off studies have reported a slight decrease in accuracy levels with higher numbers of (NPs) in the antecedent and with more distanct antecedents (Martin and McElree 2008). These effects could be due to increased interference during antecedent

\footnotetext{
${ }^{3}$ Alternative explanations for patients' failure to reactivate the antecedent include potential processing loads due to a semantic or discourse component in verb-phrase ellipsis and a possible failure to detect the covert constituent in the first place.
} 
retrieval, as more material intervenes between the antecedent and the ellipsis in these cases. For instance, a memory-based parsing model of sentence comprehension has proposed that processing difficulty results from the difficulty in retrieving phrases from memory (Lewis et al. 2006). Hence, the patterns of activations observed in Sluicing could be attributed to a complexity effect defined in terms of inference during memory retrieval. In effect, the antecedent in Sluicing would create greater interference due to the higher number of NPs in a clause compared to other antecedents (such as verb phrases in Verb-Phrase Ellipsis). Moreover, interference would differentially affect the Subject and the Object in Sluicing: the Subject is farther from the ellipsis and thus potentially subject to more interference than the Object. In this way, the processor would fail to retrieve the Subject in Sluicing due to interference.

However, in the memory-based model referred to above, interference is associated with processing costs that are detectable in reaction times. Still, neither antecedent-ellipsis distance nor number of referential NPs affects processing speed, which is inconsistent with an interference-based processing load (Martin and McElree 2008). Additionally, if the activation patterns in Sluicing are attributable to inference-induced processing costs, these factors (distance and number of referential NPs) should apply to non-clausal antecedents (e.g., verb phrases) as well. That is, non-clausal antecedents with a similarly high number of NPssome of which are distant from the ellipsis-would be expected to show interference-related processing difficulty. Yet, elliptical sentences with such long non-clausal antecedents are not processed more slowly or less accurately than elliptical sentences with shorter antecedents (Martin and McElree 2008, Exp. 4).In short, interference as an index of complexity does not straightforwardly account for the pattern of results observed in the present study.

Returning to the definition of complexity, there is one more aspect in which the antecedent in Sluicing differs from antecedents in other anaphoric relations. As mentioned above, all types of anaphora studies thus far have been reported to re-activate their syntactically-defined antecedents (noun phrases, verb phrases, etc.). Thus, the clause in Sluicing would be the first case of an antecedent not retrieved online by healthy participants. It is hence a logical possibility that the phrasal category (verb, noun phrase, verb phrase, etc.) of the antecedent in Sluicing hampered the reactivation process. This possibility relies on two important premises: 1) a clause (IP) differs in one relevant way from other phrases (and not solely in complexity as previously defined in investigations of complexity effects, e.g., number of noun phrases in the antecedent);2) there is a systemic reason for the divergent patterns between Object and Subject reactivation in Sluicing.

One hypothesis that would meet both requirements regards the involvement of inflectional nodes in the syntactic tree. In effect, only IP's implicate the inflectional nodes above the verb phrase. It is thus conceivable that retrieving information from inflectional nodes is particularly costly, perhaps to a point where the parser either elects to or cannot access this information. If this were the case, only information below the inflectional nodes would be retrieved by the parser. In ellipsis resolution, the result would be the re-activation of any antecedent lower than the inflectional nodes (e.g.: a verb phrase in VPE). In Sluicing, only the object would be reactivated; the subject would not be retrieved due to its position high in the inflectional nodes of the syntactic tree. This hypothesis is interestingly similar to the Tree Pruning Hypothesis (TPH; Friedmann and Grodzinsky 1997; Friedmann 2006). The TPH describes the functional breakdown in agrammatic production by suggesting inflectional nodes are 'pruned' from the syntactic tree, accounting for a myriad of observations on the limited output of these patients. In that sense, both hypotheses propose that inflectional nodes impute high processing costs that would lead to patients' impairments in production and the partial antecedent retrieval in Sluicing. 
Lastly, an alternative view on the lack of Subject re-activation in Sluicing reconsiders the assumption that a unit smaller than the antecedent clause is reconstructed in the elliptical clause. One possible explanation could be that the elided material is recovered as a clause, but in a syntactic form that would exclude the Subject. In effect, if the Subject is not re-accessed, how can we be sure the underlying meaning of the sluice involves the Subject? For instance, the sluice could be interpreted in the passive voice:

[8] The handyman threw a book to the programmer, but I don't know...

a. which book the handyman threw _to the programmer.

Active Voice

b. which book was thrown (by the handyman) to the programmer.

Passive Voice

Antecedent-ellipsis mismatches such as [8b] are dispreferred or more costly to process compared to their matching counterpart [8a] (Frazier and Clifton 2005; Dickey and Bunger, under review). Still, antecedent-ellipsis mismatches can be acceptable to various degrees and are in fact fairly common (Tanenhaus and Carlson 1990; Kehlar 2000; Arregui et al. 2006; Kertz 2008; see also Postdam 2007 for evidence in Sluicing). Considering that syntactic detail fades rapidly in memory (Sachs 1967), the structure of the first clause might not be available to the processor when computing an interpretation for the ellipsis. The parser might, then, reconstruct the elided clause in the truncated passive voice, a syntactic form that can grammatically exclude the agent of the action (the by-phrase in parentheses in [8b]). The passive form would naturally maintain the (syntactic and prosodic) focus on the nonsluiced object (which book) by making it the subject of the passive clause

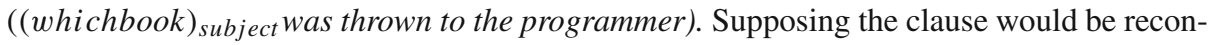
structed in this short passive form, no reactivation of the Subject handyman would be expected in the elliptical clause. This explanation is speculative, of course, but finds some support by previous research demonstrating an important role of focus in processing (see for example Carlson et al. 2009) and would reconcile the findings of this study with the literature on anaphora. The proposal also allows for full interpretation without recourse to complexity effects that the previous hypotheses suggest constrain interpretation to only non-subject NP's. This hypothesis could be tested in future work by using sentences that do not place focus on an object (e.g.: sluiced sentences with why as a remnant: The handyman threw a book to the programmer, but I don't know why ) the handym a boek to the programmer). In these conditions, the parser would be expected to reconstruct sluiced sentence in its original active form. This experiment is currently under way in our laboratory.

Clearly, more research is needed to clarify the effect, if any, of antecedent complexity on real-time ellipsis resolution and on the computation of an interpretation for a sluiced sentence. In sum, the puzzling lack of Subject re-activation, if replicated, will require careful experimentation to determine its cause. Assuming the null result holds, the absence of the Subject in the elliptical clause is problematic for syntactic and nonsyntactic accounts of ellipsis. In effect, both types of accounts would predict that the entire antecedent would be used in the computation of an interpretation for the ellipsis. Likewise for processing accounts: a cost-free copy mechanism or a content-addressable memory pointer assume there are no processing costs associated with syntactic complexity and would consequently predict that a more complex antecedent—such as a clause-could be recovered in its entirety in the elliptical clause.

In conclusion, the current study has established that the antecedent in Sluicing is reaccessed (although perhaps only partially) as the sentence unfolds, similarly to Verb-Phrase Ellipsis and other anaphoric relations. The point in time at which the ellipsis is interpreted 
in Sluicing offers additional support to the hypothesis that parallelism expectations facilitate ellipsis resolution. We believe that the patterns of activation reported here can serve as a baseline for further research investigating the effects of antecedent properties on the processing and comprehension of ellipsis.

Acknowledgments This work was supported by NIH-NIDCD DC000494 (Shapiro) as well as by the Sheila and Jeffrey Lipinsky Family Fellowship and the W.O. Dingwall Foundation Fellowship (both to Poirier). The authors thank Dr. Tracy Love for her comments on an earlier manuscript. The authors are also grateful to Roberto Gutierrez and the research assistants for their assistance with data collection.

Open Access This article is distributed under the terms of the Creative Commons Attribution Noncommercial License which permits any noncommercial use, distribution, and reproduction in any medium, provided the original author(s) and source are credited.

\section{Appendix}

See Table 2.

Table 2

S1 The ambassador loaded the bus with criminals but I don't know which bus and no one else was there to see.

$$
\text { C painting R country }
$$

S2 The actor leased a house to the lawyer but I don't know which house and I was on vacation when it happened.
C horse
R movie

S3 The skier brought a casserole to the neighbor but I don't know which casserole and I don't really care.

$$
\text { C movie R snow }
$$

S4 The jockey passed a ball to the teenager but I don't know which ball and I had a broken arm so I couldn't play.

$$
\text { C snow } \quad \text { R horse }
$$

S5 The artist poured a soda for the mentor but I don't know which soda and I was talking to Mary so I didn't notice.

$$
\text { C country R painting }
$$

S6 The astronaut sold a scooter to the gardener but I don't know which scooter and I heard the wheel fell off.

$$
\text { C ring R space }
$$

S7 The juggler served a martini to the surgeon but I don't know which martini and I already had too many to care.
C hammer
$\mathrm{R}$ ball

S8 The chemist showed an apartment to the exterminator but I don't know which apartment and no one else was home to see.

$$
\mathrm{C} \text { ball } \mathrm{R} \text { beaker }
$$

S9 The handyman threw a book to the programmer but I don't know which book and no one else seems to know.
C beaker
$\mathrm{R}$ hammer

S10 The boxer mailed a present to the teammate but I don't know which present and I couldn't be there to see it opened.

$$
\text { C space } \quad R \text { ring }
$$

S11 The author shipped a car to the new owner but I don't know which car and I was not able to afford it. 
Table 2 continued
C pool
$\mathrm{R}$ book

S12 The captain delivered a telegram to the musician but I don't know which telegram and got there too late to see.
C book
R ship

S13 The worker splashed juice on the translator but I don't know which juice and the shirt got stained.
$\mathrm{C}$ ship
$\mathrm{R}$ job

S14 The sailor sprayed confetti on the judge but I don't know which confetti and there was so many different colors that no one could tell.
$\mathrm{C}$ job
$\mathrm{R}$ boat

S15 The swimmer owed a book to the salesman but I don't know which book and I don't really care.
$\mathrm{C}$ boat
$\mathrm{R}$ pool

S16 The teacher rented a golf cart to the assistant but I don't know which golf cart and I already own one.
C building
R student

S17 The locksmith bought a boat for the reporter but I don't know which boat and I can't wait to go for a ride.
C nurse
R key

S18 The cook saved a seat for the photographer but I don't know which seat and I was already sitting across the room.
C key
$\mathrm{R}$ food

S19 The doctor wrote a letter to the hitchhiker but I don't know what letter and I couldn't care less.
$\mathrm{C}$ food
$\mathrm{R}$ nurse

S20 The architect reserved a table for the caretaker but I don't know which table and the place was so crowded so it was a good thing.
$\mathrm{C}$ student
$\mathrm{R}$ building

S21 The fisherman put a hat on the guide but I don't know which hat and I was playing soccer so I didn't notice.
C business
R tour

S22 The barber assigned a project to the dentist but I don't know which project and I was already assigned my own.
C weather
$\mathrm{R}$ teeth

S23 The builder discussed the football game with the doorman but I don't know which game and I didn't get to hear the ending score.
C teeth
R hotel

S24 The designer returned a gift to the meteorologist but I don't know which gift and I heard that it was expensive.
C hotel
$\mathrm{R}$ weather

S25 The publisher transferred a check to the entrepreneur but I don't know which check and I wasn't affected by it so I don't care.
C tour
R business

S26 The custodian gave a drink to the policeman but I don't know which drink and I think that it might have been Sprite.
C money
$\mathrm{R}$ gun

S27 The colleague handed a surfboard to the mailman but I don't know which surfboard and I heard that Bob was giving surf lessons.
C security
R letter

S28 The apprentice introduced a student to the pianist but I don't know which student and I didn't get to go.
C gun
R music 
Table 2 continued

S29 The soldier traded a baseball card with the millionaire but I don't know which card and I don't know who even likes baseball.

$$
\text { C music R money }
$$

S30 The butcher offered a sandwich to the guard but I don't know which sandwich and I already had my own.

$$
\text { C letter R security }
$$

S31 The commander promised a ring to the celebrity but I don't know which ring and I heard the answer was no.

$$
\text { C train R fame }
$$

S32 The priest placed a jacket on the king but I don't know which jacket and I hope it was warm because it was snowing outside.

$$
\text { C state R crown }
$$

S33 The veterinarian read a story to the executive but I don't know which story and I was too busy reading my own to hear.

$$
\text { C crown R office }
$$

S34 The electrician sent a package to the governor but I don't know which package and I don't really care.

$$
\text { C fame } \quad \text { R state }
$$

S35 The speaker stuck a nametag on the conductor but I don't know which nametag and I heard there ended up being a kind of mix up.

$$
\text { C office } \quad \text { R train }
$$

S36 The manufacturer stored a box for the bellboy but I don't know which box and I hope that it didn't get stolen.

$$
\text { C family R luggage }
$$

S37 The hiker loaned a bike to the clown but I don't know which bike and I heard it never got returned.

$$
\text { C sheep R nose }
$$

S38 The manager issued a key to the blacksmith but I don't know which key and I already had my own set.

$$
\text { C nose } \quad \text { metal }
$$

S39 The writer donated a check to the shepherd but I don't know which check and I don't think it was worth very much.

$$
\text { C metal R sheep }
$$

S40 The cowboy taught the game to the relative but I don't know which game and I didn't get to play.
C luggage
R family

\section{References}

Apel, J., Knoferle, P., \& Crocker, M. W. (2007). Processing parallel structure: Evidence from eye-tracking and a computational model.

Arregui, A., Clifton, C., Frazier, L., \& Moulton, K. (2006). Processing elided verb phrases with flawed antecedents: The recycling hypothesis. Journal of Memory and Language, 55(2), 232-246.

Balogh, J., Zurif, E., Prather, P., Swinney, D., \& Finkel, L. (1998). Gap-filling and end-of-sentence effects in real-time language processing: Implications for modeling sentence comprehension in aphasia. Brain and Language, 61(2), 169-182.

Bever, T. G., \& Sanz, M. (1997). Empty categories access their antecedents during comprehension: Unaccusatives in Spanish. Linguistic Inquiry, 28(1), 69-91.

Bock, K. (1986). Syntactic persistence in language production. Cognitive Psychology, 18, 355-387.

Callahan, S., Shapiro, L., \& Love, T. (submitted). Parallelism effects and verb activation: The sustained reactivation hypothesis. Journal of Psycholinguistic Research. doi:10.1007/s10936-009-9128-0.

Carlson, K., Dickey, M. W., Frazier, L., \& Clifton, C. Jr. (2007). Focus in sluicing interpretation. Paper presented at the 20th annual cuny conference on human sentence processing, La Jolla, CA. 
Carlson, K., Dickey, M. W., Frazier, L., \& Clifton, C., Jr (2009). Information structure expectations in sentence comprehension. The Quarterly Journal of Experimental Psychology, 62(1), 114-139.

Chung, S., Ladusaw, W. A., \& McCloskey, J. (1995). Sluicing and logical form. Natural Language Semantics, 3(3), 239-282.

De Goede, D., Shapiro, L. P., Wester, F., Swinney, D., \& Bastiaanse, R. (2010). The time course of verb processing in Dutch sentences. Journal of Psycholinguistic Research, 38(3), 181-199.

Dickey, M. W., \& Bunger, A. (under review). Comprehension of elided structure: Evidence from sluicing. Language and Cognitive Processes.

Frazier, L., \& Clifton, C. (1998). Comprehension of sluiced sentences. Language and Cognitive Processes, 13, 499-520.

Frazier, L., \& Clifton, C. (2001). Parsing coordinates and ellipsis: Copy. Syntax, 4(1), 1-22.

Frazier, L., \& Clifton, C. (2005). The syntax-discourse divide: Processing ellipsis. Syntax, 8(2), 121-174.

Frazier, L., \& Clifton, C., Jr. (2000). On bound variable interpretations: The LF-only hypothesis. Journal of Psycholinguistic Research, 29(2), 125-139.

Frazier, L., Munn, A., \& Clifton, C. (2000). Processing coordinate structures. Journal of Psycholinguistic Research, 29(4), 343-370.

Frazier, L., Taft, L., Roeper, T., Clifton, C., \& Ehrlich, K. (1984). Parallel structure: In sentence. Memory and Cognition, 12(5), 421-430.

Friedmann, N. (2006). Speech production in Broca's agrammatic aphasia: Syntactic tree pruning. In Y. Grodzinsky \& K. Amunts (Eds.), Broca's Region, (pp. 63-82).

Friedmann, N., \& Grodzinsky, Y. (1997). Tense and agreement in agrammatic production: Pruning the syntactic tree. Brain and Language, 56(3), 397-425.

Friedmann, N., Shapiro, L. P., Taranto, G., \& Swinney, D. (2008). The leaf fell (the leaf): The on-line processing of unaccusatives. Linguistic Inquiry, 39(3), 355-377.

Kaan, E., Wijnen, F., \& Swaab, T. Y. (2004). Gapping: Electrophysiological evidence for immediate processing of "missing" verbs in sentence comprehension. Brain and Language, 89(3), 584-592.

Kehler, A. (2000). Coherence and the resolution of ellipsis. Linguistics and Philosophy, 23(6), 533-575.

Kertz, L. (2008). Focus structure and acceptability in verb phrase ellipsis. Paper presented at the 27th west coast conference on formal linguistics, Somerville, MA.

Knoeferle, P. (2007). Eye tracking parallelism effects in coordinate constructions: the effects of "and", "but" and "while". Paper presented at the 20th annual cuny conference on human sentence processing, San Diego, CA.

Knoeferle, P., \& Crocker, M. (2010). Constituent order and semantic parallelism in on-line comprehension: eye-tracking evidence from German. Quarterly Journal of Experimental Psychology, 62(12), 2338-2371.

Koeneman, O., Bauuw, S., \& Wijnen, F. (1998). Reconstruction in VP-Ellipsis: Reflexive vs. non-reflexive predicates (Vol. Poster). In 11th annual cuny conference on human sentence processing, New Brunswick, NJ, 19-21 Mar.

Lewis, R. L., Vasishth, S., \& Van Dyke, J. A. (2006). Computational principles of working memory in sentence comprehension. Trends in Cognitive Sciences, 10(10), 447-454.

Love, T., Swinney, D., Walenski, M., \& Zurif, E. (2008). How left inferior frontal cortex participates in syntactic processing: Evidence from aphasia. Brain and Language, 107(3), 203-219.

Marantz, A. (1984). On the nature of grammatical relations. Cambridge, MA: MIT Press.

Martin, A. E., \& McElree, B. (2008). A content-addressable pointer mechanism underlies comprehension of verb-phrase ellipsis. Journal of Memory and Language, 58, 879-906.

Mauner, G., Tanenhaus, M. K., \& Carlson, G. N. (1995). A note on parallelism effects in processing deep and surface verb-phrase anaphora. Language and Cognitive Processes, 10(1), 1-12.

Nicol, J. L. (1998). Coreference processing during sentence comprehension. Massachusetts: Massachusetts Institute of Technology.

Pickering, M. J., Branigan, H. P., Cleland, A. A., \& Stewart, A. J. (2000). Activation of syntactic information during language production. Journal of Psycholinguistic Research, 29(2), 205-216.

Pickering, M. J., \& Ferreira, V. S. (2008). Structural priming: A critical review. Psychological Bulletin, $134(3), 427$.

Poirier, J., \& Shapiro, L. P. (under revision). The representation of traces: Evidence from unaccusative verbs in verb-phrase ellipsis.

Poirier, J., Shapiro, L. P., Love, T., \& Grodzinsky, Y. (2009). The on-line processing of verb-phrase ellipsis in aphasia. Journal of Psycholinguistic Research, 38(3), 237-253.

Potsdam, E. (2007). Malagasy sluicing and its consequences for the identity requirement on ellipsis. Natural Language and Linguistic Theory, 25(3), 577-613. 
Sachs, J. S. (1967). Recognition memory for syntactic and semantic aspects of connected discourse. Perception and Psychophysics, 2, 437-442.

Shapiro, L. P., \& Hestvik, A. (1995). On-line comprehension of VP-ellipsis: syntactic reconstruction and semantic influence. Journal of Psycholinguistic Research, 24(6), 517-532.

Shapiro, L. P., Hestvik, A., Lesan, L., \& Garcia, A. R. (2003). Charting the time-course of VP-ellipsis sentence comprehension: Evidence for an initial and independent structural analysis. Journal of Memory and Language, 49(1), 1-19.

Swinney, D., Ford, M., Frauenfelder, U., \& Bresnan, J. (1987). On the temporal course of gap-filling and antecedent assignment during sentence comprehension, language structure and processing. Stanford, CA: CSLI.

Tanenhaus, M. K., \& Carlson, G. N. (1990). Comprehension of deep and surface verbphrase anaphors. Language and Cognitive Processes, 5(4), 257-280.

Zurif, E., Swinney, D., Prather, P., Solomon, J., \& Bushell, C. (1993). An on-line analysis of syntactic processing in Broca's and Wernicke's aphasia. Brain and Language, 45, 448-464. 